\title{
Sphingosine-1-phosphate inhibits the adipogenic differentiation of 3T3-L1 preadipocytes
}

\author{
MYUNG-HEE MOON, JAE-KYO JEONG, YOU-JIN LEE, JAE-WON SEOL and SANG-YOUEL PARK
}

Biosafety Research Institute, College of Veterinary Medicine, Chonbuk National University, Jeonju, Jeonbuk 561-756, Republic of Korea

Received January 27, 2014; Accepted July 11, 2014

DOI: $10.3892 /$ ijmm.2014.1856

\begin{abstract}
Sphingosine-1-phosphate (S1P) is a pluripotent lipid mediator that transmits signals through G-protein-coupled receptors to control diverse biological processes. The novel biological activity of S1P in the adipogenesis of 3T3-L1 preadipocytes was identified in the present study. S1P significantly decreased lipid accumulation in maturing preadipocytes in a dose-dependent manner. In order to understand the anti-adipogenic effects of S1P, preadipocytes were treated with $\mathrm{S} 1 \mathrm{P}$, and the change in the expression of several adipogenic transcription factors and enzymes was investigated using quantitative RT-PCR. S1P downregulated the transcriptional levels of the peroxisome proliferator-activated receptor $\gamma$, CCAAT/enhancer binding proteins and adiponectin, which are markers of adipogenic differentiation. The effects of S1P on the levels of mitogen-activated protein kinase (MAPK) signals in preadipocytes were also investigated. The activation of JNK and p38 were downregulated by S1P treatment in human preadipocytes. In conclusion, the results of this study suggest that S1P alters fat mass by directly affecting adipogenesis. This is mediated by the downregulation of adipogenic transcription factors and by inactivation of the JNK and p38 MAPK pathways. Thus, selective targeting of the S1P receptors and sphingosine kinases may have clinical applications for the treatment of obesity.
\end{abstract}

\section{Introduction}

Obesity is the most common metabolic disease in developed nations and has become a global epidemic in recent years (1). Furthermore, obesity is associated with a variety of chronic diseases, including glucose intolerance, insulin resistance, dyslipidemia and hypertension. A combination of these abnor-

Correspondence to: Professor Sang-Youel Park or Professor Jae-Won Seol, Biosafety Research Institute, College of Veterinary Medicine, Chonbuk National University, 567 Baekje-daero, Deokjin-gu, Jeonju, Jeonbuk 561-756, Republic of Korea

E-mail: sypark@chonbuk.ac.kr

E-mail: jwsseol@chonbuk.ac.kr

Key words: sphingosine-1-phosphate, differentiation, adipocyte, 3T3-L1 malities is now referred to as the metabolic syndrome (2). An increase in fat mass is a result of an increase in adipocyte number and size. Cellular and molecular studies focusing on the development of obesity have shown that changes in the number of adipocytes (hyperplasia) and adipocyte size (hypertrophy) can be triggered by dietary factors $(3,4)$. The findings of a previous study indicated that an increased adipocyte number during the aging process may contribute to the increase in the incidence and severity of obesity observed in older individuals (5). Thus, hyperplasia of adipocytes may be an important factor in the development of obesity.

Adipocytes are derived from mesenchymal stem cells, which have the potential to differentiate into myoblasts, chondroblasts, osteoblasts or adipocytes (6). Adipocyte differentiation involves an elaborate network of transcription factors that regulate the expression of numerous genes responsible for the phenotype of mature adipocytes (7). Among the various transcription factors that promote preadipocyte differentiation and influence adipogenesis, peroxisome proliferator-activated receptor $\gamma(\mathrm{PPAR} \gamma)$ is considered the 'master regulator of adipogenesis' (8-10). Other adipogenic transcription factors include the CCAAT/enhancer binding proteins $(\mathrm{C} / \mathrm{EBP} \alpha, \mathrm{C} / \mathrm{EBP} \beta$ and $\mathrm{C} / \mathrm{EBP} \gamma)(5,7,11)$. These factors are necessary for the expression of adipocyte-specific genes (adiponectin) (12). These transcription factors, especially PPAR $\gamma$ and $\mathrm{C} / \mathrm{EBP} \alpha$ are regulated by the mitogen-activated protein kinase (MAPK) pathway during adipogenesis (13-15).

Sphingosine-1-phosphate (S1P) is a member of an important group of signaling sphingolipids now recognized to play a role in a diverse array of cell processes, such as apoptosis, cell motility, differentiation, and proliferation in a variety of cell types including endothelial cells, smooth muscle cells and macrophages $(16,17)$. S1P is generated by the phosphorylation of the sphingosine mediated by sphingosine kinases-1 (Sphk-1) and Sphk-2 (18). S1P exerts most of its activity as a ligand of G-protein-coupled receptors (GPCRs) (19). At present, five members of the S1P receptor family have been identified in mammals, notably S1P1-5, possessing distinct expression profiles and affinities towards $\operatorname{S1P}(20,21)$.

S1P regulates the differentiation via MAPK pathways in a variety of cell types including osteoclasts, monocytes, placental trophoblasts, myoblasts and vascular smooth muscle cells $(16,19,22-24)$. In addition, a number of studies have shown that S1P and sphingosine kinases have multifunctional 
characteristics, including a correlation with weight gain in breast cancer patients, a sensitivity to acute myeloid leukemia cells, a chemotherapy sensor in prostate cancer and enhancing sensitivity to hormone-resistant prostate cancer (25-28). ERK, p38 and JNK MAPKs are intracellular signaling pathways that play a pivotal role in numerous essential cell processes such as proliferation and differentiation $(3,13,24)$. Chemotherapy induces the downregulation of S1P by inhibiting Sphk and this decrease of circulating S1P by chemotherapy may switch S1P-mediated adipose cell stasis to adipogenesis.

In the present study, we investigated whether S1P inhibited adipocyte differentiation and regulated MAPK pathways including ERK, p38 and JNK MAPKs. S1P was found to exert novel and physiologically important biological effects on preadipocytes, acting as an anti-differentiation agent.

\section{Materials and methods}

Reagents. S1P was purchased from Cayman Chemical (Ann Arbor, MI, USA) and Sigma-Aldrich (St. Louis, MO, USA). $\mathrm{S} 1 \mathrm{P}$ was prepared as a $2 \mathrm{mM}$ solution in $0.3 \mathrm{M} \mathrm{NaOH}$ or methanol or $125 \mu \mathrm{M}$ solution in fatty acid-free bovine serum albumin, subsequently diluted in cell culture medium.

Cell culture and differentiation. 3T3-L1 cells were maintained in Dulbecco's modified Eagle's medium (DMEM) containing $10 \%$ calf serum and antibiotics $(100 \mu \mathrm{g} / \mathrm{ml}$ gentamycin and $100 \mu \mathrm{g} / \mathrm{ml}$ penicillin-streptomycin). To induce differentiation, 2-day post confluent 3T3-L1 cells were incubated in MDI induction media [DMEM containing 10\% fetal bovine serum, $0.5 \mathrm{mM}$ 3-isobutyl-1-methylxanthine (IBMX), $1 \mu \mathrm{m}$ dexamethasone and $1 \mu \mathrm{g} / \mathrm{ml}$ of insulin] for 2 days. In some experiments, $\mathrm{S} 1 \mathrm{P}(10 \mu \mathrm{M})$ was added at the time of the induction of differentiation. The AdipoRed Assay and detection of glycerol release contents were performed on day 7 .

Quantification of lipid content. Lipid content was quantified using the commercially available AdipoRed assay reagent (Lonza, Verviers, Belgium) according to the manufacturer's instructions. In brief, preadipocytes grown in 24-well plates were incubated with MDI medium or with the test compounds during the adipogenic phase and on day 6 , the culture supernatant was removed and carefully washed with $500 \mu 1$ phosphate-buffered saline (PBS). The wells were subsequently filled with $300 \mu \mathrm{l}$ PBS and $30 \mu \mathrm{l}$ of AdipoRed reagent were added followed by incubation for $10 \mathrm{~min}$ at $37^{\circ} \mathrm{C}$. The AdipoRed of the cells was photographed using a light microscope and fluorescence was measured with an excitation at $485 \mathrm{~nm}$ and an emission at $572 \mathrm{~nm}$.

Adipolysis assay. Glycerol release was measured using a commercially available Adipolysis assay kit (Cayman Chemical) according to the manufacturer's instructions. Briefly, the differentiated adipocytes in a 96-well plate were stimulated with S1P or isoproterenol solution used as a positive control for $24 \mathrm{~h}$. After stimulation, the cell culture supernatants were collected from each well and stored until use at $-20^{\circ} \mathrm{C}$. A total of $100 \mu \mathrm{l}$ of free glycerol assay reagent was added to $25 \mu \mathrm{l}$ of each supernatant. Following incubation for $15 \mathrm{~min}$ at room temperature, the absorbance was measured at $540 \mathrm{~nm}$.
Quantitative polymerase chain reaction $(R T-q P C R)$. Total RNA was extracted from 3T3-L1 cells treated with S1P using the Easy-spin ${ }^{\mathrm{TM}}$ total RNA extraction kit (Intron Biotechnology, Seoul, Korea). cDNA synthesis was carried out following the instructions of the Takara PrimeScript ${ }^{\mathrm{TM}}$ 1st Strand cDNA synthesis kit (Takara Bio, Tokyo, Japan). For the RT-qPCR, $1 \mu \mathrm{l}$ of gene primers with SYBR-Green (Bio-Rad Laboratories, Hercules, CA, USA) in $20 \mu \mathrm{l}$ of reaction volume was applied. The primer sequences used for qPCR were: PPAR $\gamma$ (forward, 5'-CGGAAGCCCTTTGG TGACTTTATG-3' and reverse, 5'-GCAGCAGGTTGTCTT GGATGTC-3'), C/EBP- $\alpha$ (forward, 5'-CGGGAACGCAAC AACATCGC-3' and reverse, 5'-TGTCCAGTTCACGGCT CAGC-3'), adiponectin (forward, 5'-TGACGGCAGCACT GGCAAG-3' and reverse, 5'-TGATACTGGTCGTAGGTGAA GAGAAC-3') $\beta$-actin (forward, 5'-TGAGAGGGAAATCG TGCGTGAC-3' and reverse, 5'-GCTCGTTGCCAATAGTGA TGACC-3'). All reactions with iTaq SYBR-Green Supermix were performed on the CFX96 real-time PCR detection system (both from Bio-Rad Laboratories).

Western blot analysis. The 3T3-L1 cells were lysed in a lysis buffer (25 mM HEPES; pH 7.4, $100 \mathrm{mM} \mathrm{NaCl,} 1 \mathrm{mM}$ EDTA, $5 \mathrm{mM} \mathrm{MgCl} 2,0.1 \mathrm{mM}$ dithiothreitol, and protease inhibitor mixture). Proteins were electrophoretically resolved on an $8-15 \%$ sodium dodecyl sulfate (SDS) gel, and immunoblotting was performed as previously described (29). Images were captured using the Fusion FX7 acquisition system (Vilber Lourmat, Eberhardzell, Germany). Densitometry of the signal bands was analyzed using Bio-1D (Vilber Lourmat) (30). The antibodies used for immunoblotting were PPAR $\gamma$ (Santa Cruz Biotechnology, Inc., Santa Cruz, CA, USA), p-JNK and p-p38 (both from Cell Signaling Technology Beverly, MA, USA) and $\beta$-actin (Sigma-Aldrich).

Statistical analysis. Data are expressed as the mean \pm standard error of the mean (SEM). Data were compared using the Student's t-test, analysis of variance (ANOVA) and Duncan test with the SAS statistical package. The results were considered significant for values of $\mathrm{P}<0.05$ or $\mathrm{P}<0.01$.

\section{Results}

S1P inhibits adipocyte differentiation of 3T3-L1 cells. Since $\mathrm{S} 1 \mathrm{P}$ regulates the differentiation of various cell types, the effect of S1P on adipocyte differentiation of the 3T3-L1 cells was investigated. Preadipocytes grown in 24-well plates were incubated with MDI media with or without S1P during the adipogenic differentiation phase. When 3T3-L1 cells differentiated over 6 days in the presence of various concentrations of $\mathrm{S} 1 \mathrm{P}$ in the adipogenic medium, a reduction in lipid accumulation was observed (Fig. 1A and B). The inhibition effect of $\mathrm{S} 1 \mathrm{P}$ was significantly detected at $0.5 \mu \mathrm{M}$ and was maximal at $50 \mu \mathrm{M}$. To confirm inhibition of triglyceride accumulation of $\mathrm{S} 1 \mathrm{P}$, we measured the triglycerides directly in 3T3-L1 cells differentiated over 6 days that were treated with S1P. S1P treatment also inhibited triglyceride accumulation during the differentiation of 3T3-L1 preadipocytes (Fig. 1C). To study the effect of S1P on lipolysis, the differentiated adipocytes were incubated with various concentrations of S1P for $24 \mathrm{~h}$, and the 
A

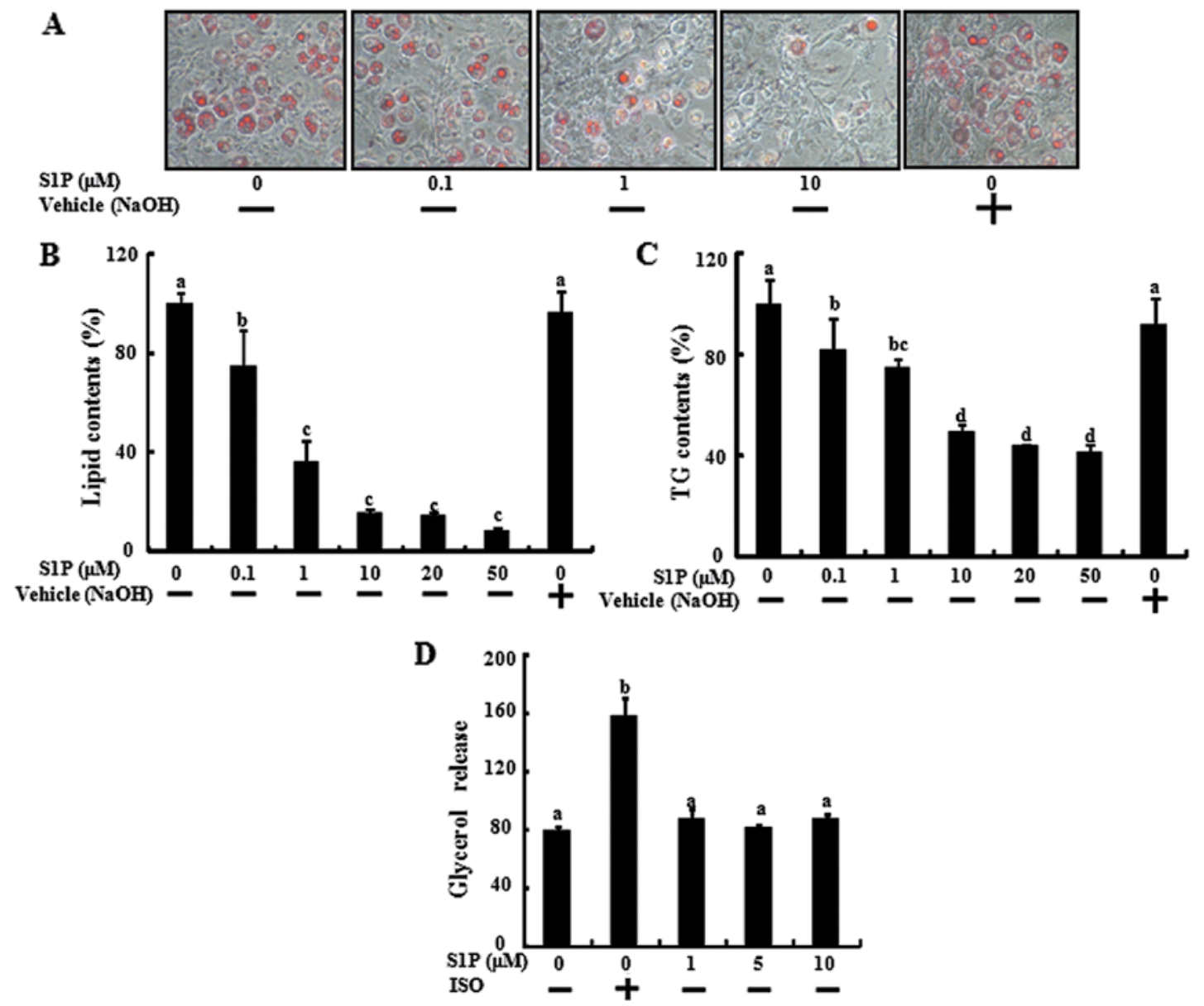

Figure 1. Sphingosine-1-phosphate (S1P) inhibits lipid accumulation during the differentiation of 3T3-L1 preadipocytes. (A) Preadipocytes were induced to differentiate with S1P of increasing concentrations and with being dissolved by vehicle including (A-C) $0.3 \mathrm{~N} \mathrm{NaOH}$ for 6 days. The AdipoRed assays were performed on day 6 and were photographed with a light microscope (x200). (B) Fluorescence was measured with excitation at $485 \mathrm{~nm}$ and emission at $572 \mathrm{~nm}$. (C) S1P inhibits triglyceride accumulation during the differentiation of 3T3-L1 preadipocytes. Preadipocytes were induced to differentiate with S1P of increasing concentrations for 6 days. The measurement of intracellular triglyceride was performed on day 6 using a triglyceride determination kit. The triglyceride level of control cells was set at $100 \%$, and triglyceride contents relative to the control are presented. (D) Differentiated adipocytes were stimulated with indicated concentrations of S1P and the concentration of glycerol in the medium was determined as described in Materials and methods. For positive controls, $3 \mu \mathrm{M}$ isoproterenol was used. (B-D) Bar graphs indicate the mean \pm standard error of the mean (SEM) from 3 different experiments. The data were analyzed using analysis of variance (ANOVA) and Duncan multiple range test $(\mathrm{P}<0.05)$. Means sharing a common alphabetical symbol do not differ significantly. Bars showing different letters indicate significant differences for each group of bars according to Duncan's test at $\mathrm{P}<0.05$.

glycerol level was determined in the medium. However, S1P did not affect glycerol release, marked to lipolysis of differentiated adipocytes (Fig. 1D), indicating that S1P inhibited lipid accumulation by blocking adipogenic differentiation, not by lipolysis of differentiated adipocytes.

We investigated whether the inhibition effects of S1P are maintained in various dissolving solutions of S1P, including fatty acid-free albumin and methyl alcohol. We tested the adipogenic differentiation, lipid contents and glycerol release assay using S1P dissolved in methyl alcohol $(\mathrm{MeOH})$ and fatty acid-free albumin stock solution in 3T3-L1 preadipocytes (Fig. 2). S1P dissolved in $\mathrm{MeOH}$ and in fatty acid-free albumin inhibited lipid accumulation but did not affect the glycerol release. The results demonstrated that S1P inhibited lipid accumulation by inhibiting adipogenic differentiation without regulating the lipolysis of adipocyte in 3T3-L1 cells.

SIP downregulates the transcriptional factor, PPAR $\gamma$, involved in adipocyte differentiation. To confirm the inhibitory effects of SIP on adipogenic differentiation, the mRNA levels of biochemical markers of differentiation (PPAR $\gamma, \mathrm{C} / \mathrm{EBP} \alpha$ and adiponectin) were determined (Figs. 3 and 4). When the 3T3-L1 preadipocytes differentiated with MDI treatment, the mRNA levels of the biochemical markers of differentiation increased compared to the control. However, S1P treatment led to a significant reduction by increasing the dose of S1P in the mRNA level of PPAR $\gamma, \mathrm{C} / \mathrm{EBP} \alpha$ and adiponectin (Figs. 3A, 4A and 4B). In the case of PPAR $\gamma$, the protein expression was also decreased by S1P (Fig. 3B).

S1P is interconvertible with ceramide and it is a critical mediator of apoptosis. Therefore, we investigated the cell number of 3T3-L1 cells during the differentiation in the presence of $10 \mu \mathrm{M}$ of S1P. At $24 \mathrm{~h}$ after MDI induction, mitosis occurred, thus the cell numbers were doubled (31). Consistent with results of that study, in the present study, the cell numbers of the MDI induction were doubled in the control and those of the S1P treatment were similar to the control (Fig. 3C). These results demonstrated that S1P exhibits anti-adipogenic activity through downregulation of the transcription factors involved in adipocyte differentiation. 
A
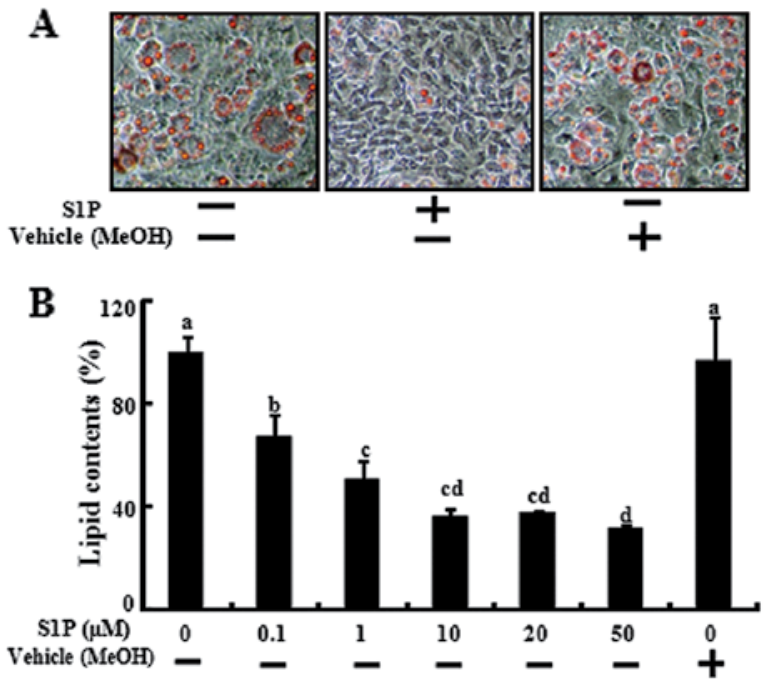

C

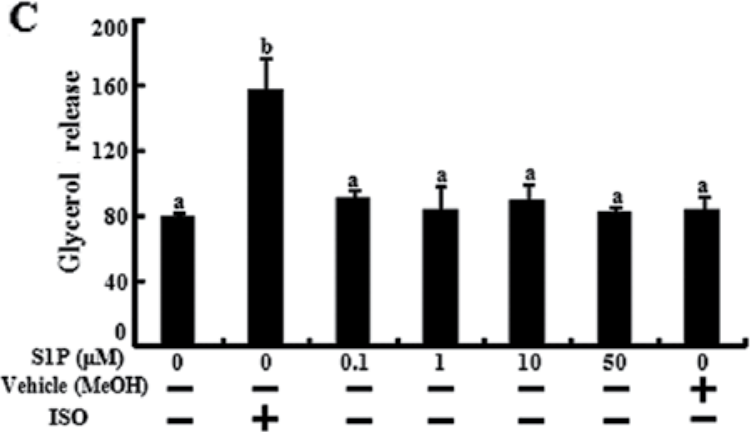

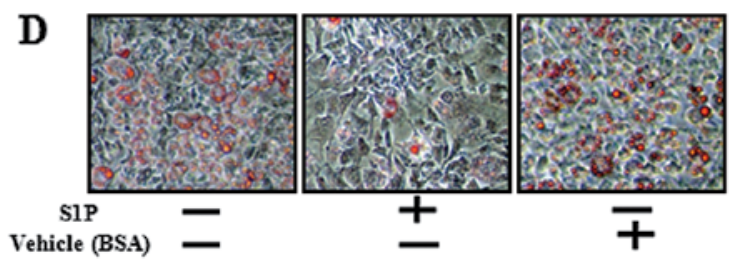
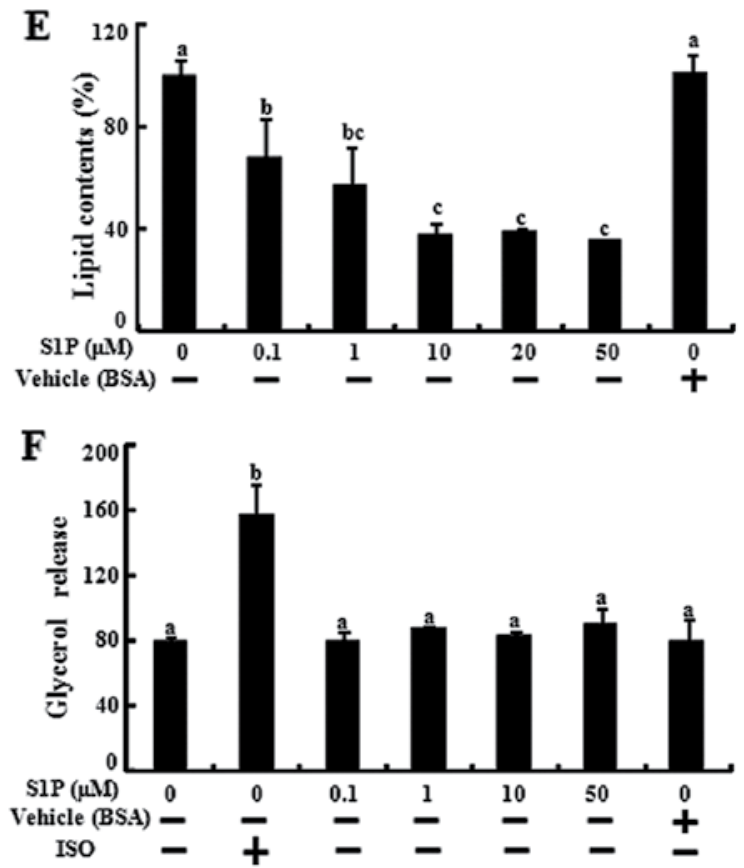

Figure 2. Sphingosine-1-phosphate (S1P) inhibits lipid accumulation during the differentiation of 3T3-L1 preadipocytes. (A and D) Preadipocytes were induced to differentiate with S1P of increasing concentrations and with being dissolved by different vehicles including (A-C) methanol (MeOH) and (D-F) $4 \mathrm{mg} / \mathrm{ml}$ of fatty acid-free bovine serum albumin solution for 6 days. The AdipoRed assays were performed on day 6 and were photographed with a light microscope (x200). (B and E) Fluorescence was measured with excitation at $485 \mathrm{~nm}$ and emission at $572 \mathrm{~nm}$. (C and F) Differentiated adipocytes were stimulated with indicated concentrations of S1P and the concentration of glycerol in the medium was determined as described in Materials and methods. For positive controls, $3 \mu \mathrm{M}$ isoproterenol was used. Bar graphs indicate the mean \pm standard error of the mean (SEM) from 3 different experiments. The data were analyzed using analysis of variance (ANOVA) and the Duncan multiple range test $(\mathrm{P}<0.05)$. Means sharing a common alphabetical symbol do not differ significantly. Bars showing different letters indicate significant differences among each group of bars according to the Duncan's test at $\mathrm{P}<0.05$.

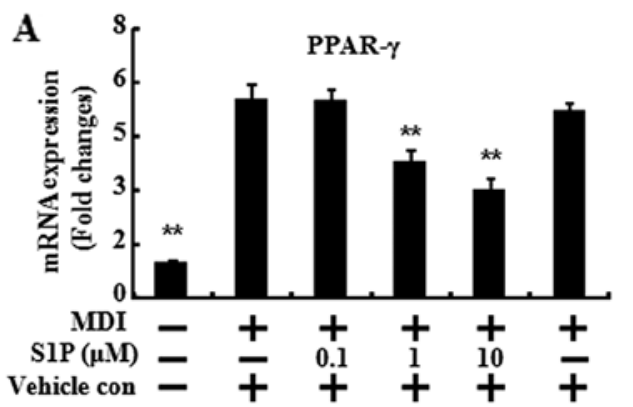

B

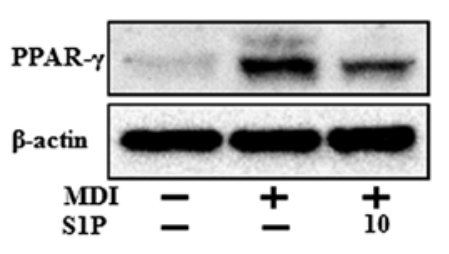

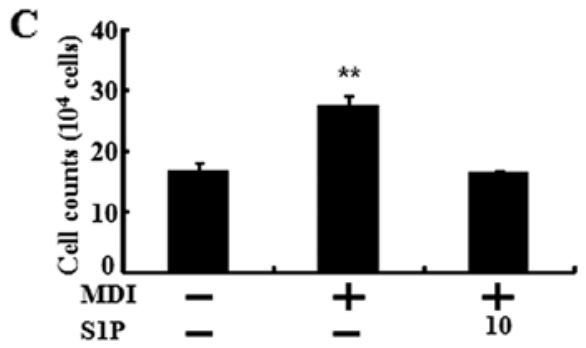

Figure 3. Sphingosine-1-phosphate (S1P) downregulates the expression of peroxisome proliferator-activated receptor $\gamma$ (PPAR $\gamma$ ), main transcriptional factors for adipogenesis. (A) Preadipocytes were induced to differentiate with S1P (1 and $10 \mu \mathrm{M})$ and harvested at day 7 during the differentiation period. (A) The mRNA expression of PPAR $\gamma$ was analyzed by RT-qPCR. Values are the mean \pm standard error of the mean (SEM) of data from 3 separate experiments. Each experiment was performed in triplicate. ${ }^{*} \mathrm{P}<0.05$ and ${ }^{* *} \mathrm{P}<0.01$ show significant differences between control and each treatment group. (B) Preadipocytes were induced to differentiate with S1P $10 \mu \mathrm{M}$ for 6 days. At day 6, the protein levels of PPAR $\gamma$ were analyzed by western blot. (C) Day 0 post-confluent 3T3-L1 preadipocytes were treated with MDI induction media and/or $10 \mu \mathrm{M}$ of S1P. After $24 \mathrm{~h}$, the cell number was determined by the trypan blue exclusion test, and the absolute cell number was plotted. The experiments were repeatedly performed to confirm the results. The data were analyzed using analysis of variance (ANOVA) and Duncan multiple range test $(\mathrm{P}<0.05)$.

S1P mediates its action on MAPK pathways via the S1P2 receptor subtype. It is well known that preadipocyte differentiation involves the activation of several key signaling pathways such as JNK1/2 and p38 MAPK (13). To gain insight into the molecular mechanisms responsible for the observed biological effects of S1P, the ability of the sphingolipid to inactivate these protein kinases was examined. The MDI containing adipocyte differentiation cocktail induced the phosphorylation of JNK1/2 
A

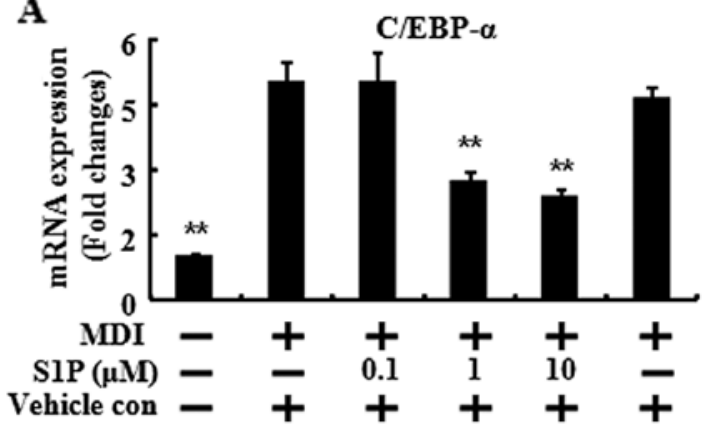

B

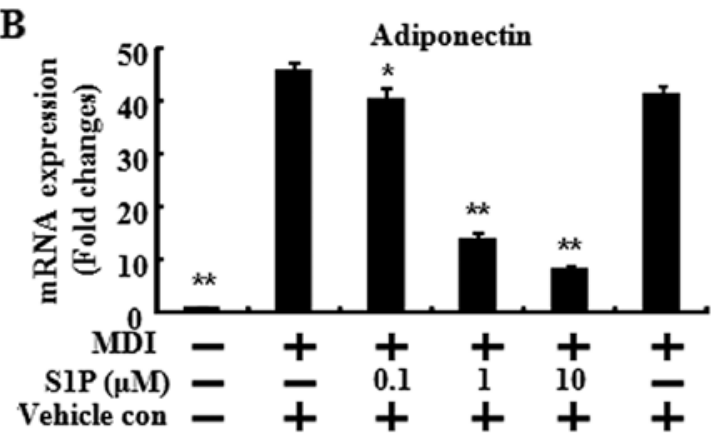

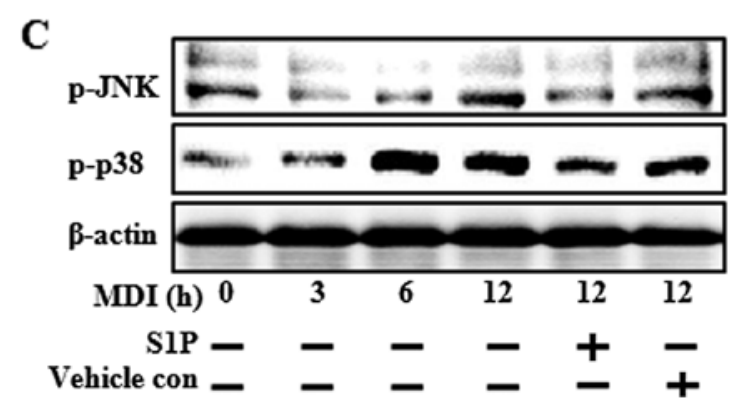

Figure 4. Sphingosine-1-phosphate (S1P) downregulates the expression of transcriptional factors for adipogenesis and adipocyte-specific gene. (A) Preadipocytes were induced to differentiate with $\mathrm{S} 1 \mathrm{P}(1$ and $10 \mu \mathrm{M})$ and harvested at day 7 during the differentiation period. The mRNA expression of (A) CCAAT/enhancer binding protein $\alpha(\mathrm{C} / \mathrm{EBP} \alpha)$ and $(\mathrm{B})$ adiponectin were analyzed by RT-qPCR. Values are mean \pm standard error of the mean (SEM)) of data from 3 separate experiments. Each experiment was performed in triplicate. " $\mathrm{P}<0.05$ and ${ }^{* *} \mathrm{P}<0.01$ show significant differences between the control and each treatment group. (C) Preadipocytes were induced to differentiate with or without S1P $10 \mu \mathrm{M}$ harvested at $12 \mathrm{~h}$ during the differentiation period. Preadipocytes were induced to differentiate with or without S1P $10 \mu \mathrm{M}$ harvested at 3,6 and $12 \mathrm{~h}$ during the differentiation period. The phosphorylation of JNK and p38 were analyzed by immunoblotting with antibodies specific for p-JNK and p-p38. The experiments were repeatedly performed to confirm the results..

and $38 \mathrm{MAPK}$, at 12, 6 and $3 \mathrm{~h}$, respectively, after MDI addition (Fig. 4C). However, $10 \mu \mathrm{M}$ of S1P decreased the phosphorylation of JNK1/2 and p38 MAPK at $12 \mathrm{~h}$ after the addition of MDI. Taken together, these results showed that S1P inhibited the adipocyte differentiation and lipid accumulation, and the inhibition effects were mediated by the downregulation of transcription factors and by inactivation of the MAPK signals.

\section{Discussion}

Excessive adipose tissue accumulation is a key factor leading to insulin resistance, type 2 diabetes, hyperlipidemia and an increased risk of cardiovascular disease. Obesity is no longer considered to be only a cosmetic problem, but is associated with an increased risk for the development of numerous adverse health conditions $(1,6)$. The recruitment of new fat cells in adipose tissue requires the differentiation of preadipocytes into adipocytes (adipogenesis), a process closely controlled by the transcription factors PPAR $\gamma$ and $\mathrm{C} / \mathrm{EBP} \alpha(32,33)$.

Studies on the effects of S1P on cell differentiation are available. S1P acts as a regulator of osteoclast differentiation (22) as well as myogenic differentiation $(24,34)$. S1P and the S1P1 receptor are associated with angiogenic differentiation of vascular endothelial cells (35). Although S1P has been demonstrated to promote the differentiation of endothelial cells and myocytes, the ability of S1P to affect cell differentiation appears to be dependent on the cell type. In placental trophoblasts and human monocytes, S1P shows anti-differentiating effects. S1P inhibits the differentiation of cytotrophoblasts into syncytiotrophoblasts through a G(i)-coupled S1P receptor interaction (16). In addition, S1P interferes with the differentiation of human monocytes into competent dendritic cells (23). Results of previous studies are in concordance with our results showing that S1P inhibits the differentiation of preadipocytes into adipocytes in 3T3-L1 cells (Figs. 1 and 2).

S1P levels inside cells are closely regulated by the balance between its synthesis by sphingosine kinases and degradation. S1P is interconvertible with ceramide, which is a critical mediator of apoptosis. In the present study, a high dose of S1P was utilized to determine the anti-adipogenic effect of S1P. To verify whether the high dose of S1P can be converted to ceramide and can equally activate the S1P receptors, further experiments are required in future studies. The ceramide itself served as an important second messenger in various stress responses and growth mechanisms (36). While S1P functions mainly via GPCR, ceramide and its metabolite appears to bind directly to targets (36).

The p38 and JNK MAPKs are intracellular signaling pathways that play a pivotal role in numerous essential cell processes such as proliferation and differentiation $(3,13,24)$. MAPKs are activated by a large variety of stimuli and one of their major functions is to connect cell surface receptors to transcription factors in the nucleus, which consequently triggers long-term cell responses (13). Previously, it was established that, the MAPK signaling pathway regulates the expression of PPAR $\gamma$ and $\mathrm{C} / \mathrm{EBP} \alpha$ during adipogenesis in preadipocytes (37). A well-known stimulus that affects the MAPK signaling pathways is S1P. The results of this study have shown that S1P inhibited MDI-induced phosphorylation of p38 and JNK1/2 (Fig. 4C). 
When induced to differentiate, growth-arrested 3T3-L1 preadipocytes synchronously re-enter the cell cycle and undergo mitotic clonal expansion (MCE). MCE is a prerequisite for the differentiation of 3T3-L1 preadipocytes into adipocytes (31). Consistent with that study, our results show that the number of cells at $24 \mathrm{~h}$ after MDI induction was increased whereas the addition of S1P significantly decreased cell populations (Fig. 3C). The results suggest that S1P inhibited the first round of mitosis, thereby preventing the expression of adipogenic regulator genes.

In conclusion, the results of this study have shown that exposure of preadipocytes to S1P inhibited their differentiation into adipocytes, as confirmed by a reduction in triglyceride accumulation and a reduction in the expression of adipocyte specific genes. Therefore, S1P functioned as an anti-adipogenic compound. The results also suggest that the adipogenic transcription factors and various MAPK pathways are a potential therapeutic target for obesity.

\section{Acknowledgements}

This study was supported by a grant from the National Research Foundation of Korea (NRF), funded by the Korean government (2013R1A1A2063931).

\section{References}

1. Lei F, Zhang XN, Wang W, et al: Evidence of anti-obesity effects of the pomegranate leaf extract in high-fat diet induced obese mice. Int J Obes (Lond) 31: 1023-1029, 2007.

2. Boyle KB, Hadaschik D, Virtue S, et al: The transcription factors Egr1 and Egr2 have opposing influences on adipocyte differentiation. Cell Death Differ 16: 782-789, 2009.

3. Kim KJ, Lee OH and Lee BY: Fucoidan, a sulfated polysaccharide, inhibits adipogenesis through the mitogen-activated protein kinase pathway in 3T3-L1 preadipocytes. Life Sci 86: 791-797, 2010.

4. Gregoire FM, Smas CM and Sul HS: Understanding adipocyte differentiation. Physiol Rev 78: 783-809, 1998.

5. Yanagiya T, Tanabe A and Hotta K: Gap-junctional communication is required for mitotic clonal expansion during adipogenesis. Obesity (Silver Spring) 15: 572-582, 2007.

6. Rayalam S, Della-Fera MA and Baile CA: Phytochemicals and regulation of the adipocyte life cycle. J Nutr Biochem 19: 717-726, 2008

7. Lee H, Lee YJ, Choi H, Ko EH and Kim JW: Reactive oxygen species facilitate adipocyte differentiation by accelerating mitotic clonal expansion. J Biol Chem 284: 10601-10609, 2009.

8. Wakabayashi K, Okamura M, Tsutsumi S, et al: The peroxisome proliferator-activated receptor gamma/retinoid $\mathrm{X}$ receptor alpha heterodimer targets the histone modification enzyme PR-Set7/ Setd 8 gene and regulates adipogenesis through a positive feedback loop. Mol Cell Biol 29: 3544-3555, 2009.

9. Tontonoz P and Spiegelman BM: Fat and beyond: the diverse biology of PPARgamma. Annu Rev Biochem 77: 289-312, 2008.

10. Rosen ED and MacDougald OA: Adipocyte differentiation from the inside out. Nat Rev Mol Cell Biol 7: 885-896, 2006.

11. Nerurkar PV, Lee YK and Nerurkar VR: Momordica charantia (bitter melon) inhibits primary human adipocyte differentiation by modulating adipogenic genes. BMC Complement Altern Med 10: 34, 2010.

12. Xing Y, Yan F, Liu Y and Zhao Y: Matrine inhibits 3T3-L1 preadipocyte differentiation associated with suppression of ERK1/2 phosphorylation. Biochem Biophys Res Commun 396: 691-695, 2010 .

13. Bost F, Aouadi M, Caron L and Binétruy B: The role of MAPKs in adipocyte differentiation and obesity. Biochimie 87: 51-56, 2005.

14. Alonso-Vale MI, Peres SB, Vernochet C, Farmer SR and Lima FB: Adipocyte differentiation is inhibited by melatonin through the regulation of $\mathrm{C} / \mathrm{EBPbeta}$ transcriptional activity. $\mathrm{J}$ Pineal Res 47: 221-227, 2009.
15. Chen TH, Chen WM, Hsu KH, Kuo CD and Hung SC: Sodium butyrate activates ERK to regulate differentiation of mesenchymal stem cells. Biochem Biophys Res Commun 355: 913-918, 2007.

16. Johnstone ED, Chan G, Sibley CP, Davidge ST, Lowen B and Guilbert LJ: Sphingosine-1-phosphate inhibition of placental trophoblast differentiation through a $\mathrm{G}(\mathrm{i})$-coupled receptor response. J Lipid Res 46: 1833-1839, 2005.

17. Goetzl EJ, Wang W, McGiffert C, Liao JJ and Huang MC: Sphingosine 1-phosphate as an intracellular messenger and extracellular mediator in immunity. Acta Paediatr Suppl 96: 49-52, 2007.

18. He X, H'ng S C, Leong DT, Hutmacher DW and Melendez AJ: Sphingosine-1-phosphate mediates proliferation maintaining the multipotency of human adult bone marrow and adipose tissuederived stem cells. J Mol Cell Biol 2: 199-208, 2010.

19. Schüppel M, Kürschner U, Kleuser U, Schäfer-Korting M and Kleuser B: Sphingosine 1-phosphate restrains insulin-mediated keratinocyte proliferation via inhibition of Akt through the S1P2 receptor subtype. J Invest Dermatol 128: 1747-1756, 2008

20. Bieberich E: There is more to a lipid than just being a fat: sphingolipid-guided differentiation of oligodendroglial lineage from embryonic stem cells. Neurochem Res 36: 1601-1611, 2011.

21. Pyne S and Pyne NJ: Sphingosine 1-phosphate signalling in mammalian cells. Biochem J 349: 385-402, 2000.

22. Ryu J, Kim HJ, Chang EJ, Huang H, Banno Y and Kim HH: Sphingosine 1-phosphate as a regulator of osteoclast differentiation and osteoclast-osteoblast coupling. EMBO J 25: 5840-5851, 2006.

23. Martino A, Volpe E, Auricchio G, et al: Sphingosine 1-phosphate interferes on the differentiation of human monocytes into competent dendritic cells. Scand J Immunol 65: 84-91, 2007.

24. Donati C, Meacci E, Nuti F, Becciolini L, Farnararo M and Bruni P: Sphingosine 1-phosphate regulates myogenic differentiation: a major role for S1P2 receptor. FASEB J 19: 449-451, 2005.

25. Pchejetski D, Nunes J, Sauer L, et al: Circulating sphingosine-1phosphate inversely correlates with chemotherapy-induced weight gain during early breast cancer. Breast Cancer Res Treat 124: 543-549, 2010.

26. Bonhoure E, Pchejetski D, Aouali N, et al: Overcoming MDR-associated chemoresistance in HL-60 acute myeloid leukemia cells by targeting sphingosine kinase-1. Leukemia 20: 95-102, 2006.

27. Pchejetski D, Golzio M, Bonhoure E, et al: Sphingosine kinase-1 as a chemotherapy sensor in prostate adenocarcinoma cell and mouse models. Cancer Res 65: 11667-11675, 2005.

28. Sauer L, Nunes J, Salunkhe V, et al: Sphingosine kinase 1 inhibition sensitizes hormone-resistant prostate cancer to docetaxel. Int J Cancer 125: 2728-2736, 2009.

29. Moon MH, Jeong JK, Seo JS, et al: Bisphosphonate enhances TRAIL sensitivity to human osteosarcoma cells via death receptor 5 upregulation. Exp Mol Med 43: 138-145, 2011.

30. Seo JS, Moon MH, Jeong JK, et al: SIRT1, a histone deacetylase, regulates prion protein-induced neuronal cell death. Neurobiol Aging 33: 1110-1120, 2012.

31. Tang QQ, Otto TC and Lane MD: Mitotic clonal expansion: a synchronous process required for adipogenesis. Proc Natl Acad Sci USA 100: 44-49, 2003.

32. Simon MF, Daviaud D, Pradère JP, et al: Lysophosphatidic acid inhibits adipocyte differentiation via lysophosphatidic acid 1 receptor-dependent down-regulation of peroxisome proliferatoractivated receptor gamma2. J Biol Chem 280: 14656-14662, 2005.

33. Fu L, Tang T, Miao Y, Zhang S, Qu Z and Dai K: Stimulation of osteogenic differentiation and inhibition of adipogenic differentiation in bone marrow stromal cells by alendronate via ERK and JNK activation. Bone 43: 40-47, 2008

34. Meacci E, Cencetti F, Donati C, et al: Down-regulation of EDG5/S1P2 during myogenic differentiation results in the specific uncoupling of sphingosine 1-phosphate signalling to phospholipase D. Biochim Biophys Acta 1633: 133-142, 2003.

35. Liu Y, Wada R, Yamashita T, et al: Edg-1, the G protein-coupled receptor for sphingosine-1-phosphate, is essential for vascular maturation. J Clin Invest 106: 951-961, 2000.

36. Chalfant CE and Spiegel S: Sphingosine 1-phosphate and ceramide 1-phosphate: expanding roles in cell signaling. J Cell Sci 118: 4605-4612, 2005.

37. Prusty D, Park BH, Davis KE and Farmer SR: Activation of MEK/ ERK signaling promotes adipogenesis by enhancing peroxisome proliferator-activated receptor gamma (PPARgamma ) and C/ EBPalpha gene expression during the differentiation of 3T3-L1 preadipocytes. J Biol Chem 277: 46226-46232, 2002. 